\section{P43 SAFETY OF PRE-OPERATIVE LUNG FUNCTION TESTING IN PATIENTS WITH A THORACIC OR THORACO-ABDOMINAL AORTIC ANEURYSM}

doi:10.1136/thoraxjnl-2012-202678.184

PV Joshi, P Bourke, S Martin, WLG Oldfield. Imperial College Healthcare NHS Trust, London, United Kingdom

Introduction Lung function testing is a useful component of the pre-operative assessment of patients being considered for major surgery. Current national and international guidelines suggest that the presence of a thoracic aortic aneurysm is a contra-indication to testing as the major swings in intra-thoracic pressure that occur during lung function testing may precipitate rupture of the aneurysmal sac. The evidence base for this recommendation is minimal and based on expert opinion only.

Aims To audit the use of pulmonary function testing in the preoperative assessment of patients with a thoracic or thoracoabdominal aortic aneurysm.

Methods All patients who were referred to a tertiary vascular surgical unit for elective or urgent repair of a thoracic or thoracoabdominal aortic aneurysm over a 60 month period (January 2005 until January 2010) were included in the audit. Patients who were referred as an emergency, or assessed for surgery but not treated, were excluded. Pre-operative pulmonary function studies for these patients were reviewed, particularly any comments by the Physiologist. Incident reports for the Respiratory Physiology Laboratory during this period were also requested.

Results 183 patients were referred and subsequently operated on. Of these, lung function test results were not available for 11 patients. Of the remaining 172 patients, no adverse incidents (either immediate or late) were recorded for 171 patients. One patient complained of pain during tests but the nature of this pain, or any associated adverse event, was not documented. This patient proceeded to elective repair of the aneurysm 8 days after testing. There were no clinical incident forms submitted from the Respiratory Physiology Laboratory during this period associated with patients undergoing an aortic aneurysm repair workup.

Conclusions Our findings suggest that the pre-operative assessment of patients being considered for aortic aneurysm repair can safely include lung function testing. We suggest collating data from other vascular surgical units to confirm our observations and thus inform future guideline development.

\section{P44 HOW LONG IS SHORT TERM OXYGEN THERAPY? HOME OXYGEN PRESCRIPTIONS POST HOSPITALISATION}

doi:10.1136/thoraxjnl-2012-202678.185

JL Gallagher, G McKernan, L Slater, A DeRamon. Warrington and Halton Hospitals NHS Foundation Trust, Warrington, United Kingdom

Introduction Long-term oxygen therapy (LTOT) has been shown to improve survival in some patients with chronic obstructive pulmonary disease (COPD); generalisation to other groups of hypoxic patients is less clear. The British Thoracic Society recommend LTOT should be prescribed to hypoxic patients after appropriate assessment during a period of clinical stability. ${ }^{1}$ In practise LTOT is frequently prescribed following hospitalisation. This form of oxygen therapy has been termed short-term oxygen therapy (STOT). We sought to audit our practise of prescribing home oxygen therapy to hypoxic patients following hospital admission with an exacerbation of a respiratory disorder.

Method We performed a retrospective case note audit of 30 patients [13 male] commenced on home oxygen therapy at discharge over a 6 month period recording patient demographics, smoking status, underlying diagnosis, compliance with BTS oxygen prescribing guidelines and the outcome of follow-up.
Results The mean age was 71 years (48-91yrs); $17 \%$ current smokers, $73 \%$ ex-smokers. COPD was the most common diagnosis in $80 \%$ (24/30); the mean FEV1 1.1L (52\% predicted). No definitive diagnosis was documented in 10\% (3/30) with 10\% diagnosed with sarcoidosis, idiopathic pulmonary fibrosis or lung cancer. $64 \%$ $(19 / 30)$ of patients met BTS LTOT criteria, $(80 \%$ (19/24) with COPD), 33\% (10/30) did not and 3\% (1/30) were prescribed palliative oxygen. $93 \%$ of patients $(27 / 29)$ were followed up at 3 months. The mean oxygen flow rate required was $1.4 \mathrm{~L} / \mathrm{min}$; oxygen flow rate was altered in $63 \%$. The overwhelming majority of our patients, $90 \%(26 / 29)$, continued with LTOT after reassessment at 3 months and $79 \%(23 / 29)$ at 6 months.

Conclusion Our audit shows that the majority of patients commenced on oxygen therapy post hospitalisation met the criteria for LTOT and continued to require oxygen at 3 and 6 months; this is a higher figure than quoted in the majority of the literature. This suggests our patients are presenting late with underlying chronic lung disease; we need to strive to identify these patients earlier. There is still a lack of evidence-based guidelines for STOT and further research in this area is needed.

\section{References}

1. BTS Home Oxygen Service in England and Wales. January 2006.

\section{P45 AUDIT OF OXYGEN MANAGEMENT ON NINE MEDICAL WARDS USING ELECTRONIC PRESCRIBING SYSTEMS}

doi:10.1136/thoraxjnl-2012-202678.186

AA Nasir, BR O'Driscoll Salford. Royal University Hospital, Salford, UK

Nationwide UK audits by the British Thoracic Society have shown that more than half of UK hospital patients who are using oxygen do not have a prescription for this drug (Thorax. 2011; 66:734). We have audited the prescription and monitoring of oxygen use amongst 347 patients on nine medical wards at our 800 bedded teaching hospital which has a policy of prescribing a target oxygen saturation range for all patients on admission to hospital, using an "admissions bundle" within the electronic prescribing system.

$97 \%$ of these medical patients had an oxygen saturation range prescribed in the electronic record and a further 1\% had a target range specified on bedside documents. $79 \%$ of prescriptions were for a target range of $94-98 \%, 20 \%$ were for a range of $88-92 \%$ and $1 \%$ were for patient-specific target ranges. $10 \%$ of medical patients were receiving oxygen at the time of this audit.

$92 \%$ of all patients were within their specified target range, $3 \%$ were above the target range (all of them on oxygen therapy) and $2 \%$ were below their target range (all of them breathing air at the time of audit). In 17 of 19 cases where patients were outside their target range, no action was taken by those making the observations. A particular concern in this audit was that $4 \%$ of patients had a different target saturation range on the bedside observation chart compared with the prescribed range. This led to some of the above errors.

$14 \%$ of medical patients had risk factors for type 2 respiratory failure. Of the 26 patients who had blood gases taken on the day of audit, $35 \%$ had type 1 respiratory failure and $27 \%$ had type 2 failure.

This audit has shown that electronic prescribing systems with an "admissions bundle" can achieve target prescriptions for oxygen for over $95 \%$ of medical patients. However there were discrepancies with the bedside charts in $4 \%$ of cases, a problem that will be solved when bedside observations become electronic in future. There are also concerns that appropriate action was not taken in many cases where patients were outside the target range. 